\title{
Evaluation of Stress Systems by Applying Noninvasive Methodologies: Measurements of Neuroimmune Biomarkers in the Sweat, Heart Rate Variability and Salivary Cortisol
}

\author{
Andrea H. Marques Marni N. Silverman Esther M. Sternberg
}

National Institute of Mental Health/NIH, Rockville, Md., USA

\section{Key Words}

Cytokines $\cdot$ Neuropeptides $\cdot$ Cardiovascular system $\cdot$ HPA axis

\begin{abstract}
The two main arms of the stress system include the autonomic nervous system (ANS) and the hypothalamic-pituitary-adrenal (HPA) axis. These two neural stress systems coordinate the response of many other physiological systems to a stressor, including the immune and cardiovascular systems, bringing the body back to homeostasis. The nervous and immune systems communicate with each other in a bidirectional manner. In this review, we will discuss the use of noninvasive methods to evaluate the immune system, ANS and HPA axis. Collection of sweat and saliva, and measurement of heart rate variability are noninvasive methods that can be applied to evaluate neuroimmune interactions. Recently, we validated a new methodology to simultaneously evaluate a large array of neural and immune biomarkers in sweat, collected through cutaneous sweat patches and measured by recycling immunoaffinity chromatography. Noninvasive and ambulatory methodologies of biomarker collection can overcome several limitations intrinsic to invasive methods, such as reducing the stress triggered by collection
\end{abstract}

itself and allowing a wider application to field and community-based settings. Ultimately, simultaneous evaluation of neural and immune systems with noninvasive techniques will help elucidate the underlying interactions of these systems and their role in disease susceptibility and progression of stress-related disorders.

Copyright $\odot 2010$ S. Karger AG, Basel

\section{Introduction}

Two main pathways by which psychological stress is relayed to the body are the hypothalamic-pituitary-adrenal (HPA) axis (neuroendocrine route, resultant release of glucocorticoids - cortisol) and the sympathetic nervous system (SNS, neural route, resultant release of catecholamines - noradrenaline/adrenaline - and neuropeptides). Dysregulation of either of these stress systems can lead to dysregulation of multiple other physiological systems, including the immune and cardiovascular systems, metabolic function and behavior, leading to a maladaptive response to stress (fig. 1).

The nervous and immune systems communicate with each other in a bidirectional manner [1, 2]. Indeed, dysregulation of neural-immune interactions are described

\section{KARGER}

(C) 2010 S. Karger AG, Basel

Fax +4161306 1234

E-Mail karger@karger.ch

www.karger.com
Accessible online at:

www.karger.com/nim
Esther M. Sternberg, MD, Integrative Neural Immune Program and Chief Section on Neuroendocrine Immunology and Behavior National Institute of Mental Health/NIH, 5625 Fishers Lane MSC 9401, Rm. 4N-13B

Rockville, MD 20892-9401 (USA)

Tel. +1 301402 2773, Fax +1 301496 6095, E-Mail sternbee@mail.nih.gov 


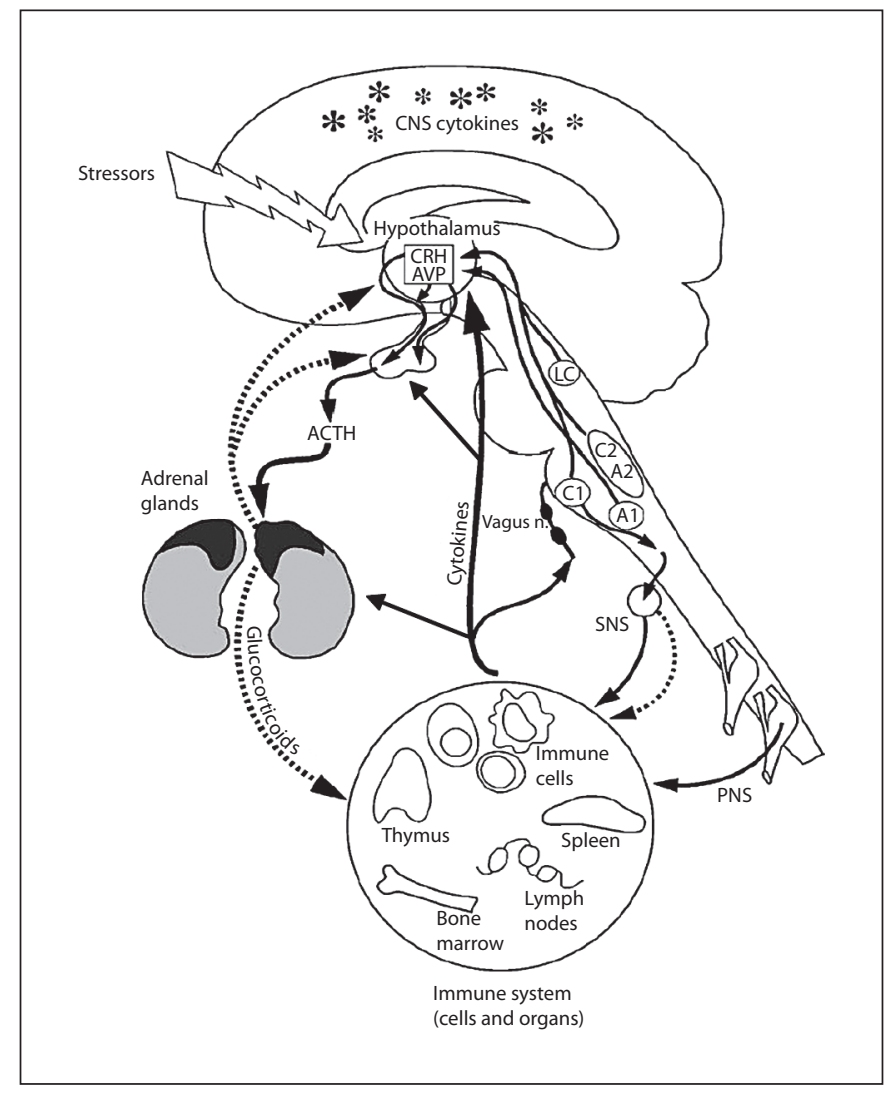

Fig. 1. Diagram of the routes of communication between the brain and immune system, including the HPA axis, autonomic nervous system, and cytokine interactions with both of these systems. CNS = Central nervous system; SNS = sympathetic nervous system; PNS = peripheral nervous system; $\mathrm{CRH}=$ corticotropin-releasing hormone; AVP = arginine vasopressin; ACTH = adrenocorticotropic hormone. Reprinted with modifications by permission from Webster et al. [9].

in many stress-related disorders including inflammatory, autoimmune, metabolic and cardiovascular disease, as well as psychiatric disorders. Simultaneous evaluation of stress and immune systems with noninvasive, and thus non-stress-inducing, techniques will help to elucidate the underlying interactions of these systems and their role in disease susceptibility and progression of stress-related disorders. In this review, we will briefly discuss noninvasive methodologies that can be simultaneously applied in order to evaluate neural and immune system biomarkers in sweat, the ANS by measuring heart rate variability (HRV) and the HPA axis by measuring cortisol in saliva.

\section{Neuroimmune Biomarker Evaluation}

One noninvasive and nonstressful approach to evaluating neural and immune systems is through collection of sweat. We have validated a methodology to simultaneously evaluate a large array of neural and immune biomarkers in sweat, collected through cutaneous sweat patches.

The cutaneous sweat patch is commercially available and FDA approved. It is easily applied at any time of day and can be worn for an extended period of time with minimal discomfort. In addition, since sweat collection is over a 24-hour period, rather than a single time point, the use of the sweat patch may overcome circadian variability exhibited by analytes of interest. In our validation study, subjects were instructed to apply two sweat patches at $8 \mathrm{a} . \mathrm{m}$. on the day before coming into the laboratory for blood collection, and wore them for $24 \mathrm{~h}$. On the next day, blood samples were obtained at 8 a.m., after an overnight fast, prior to the removal of the sweat patches. The sweat patch was placed in a zip lock bag and stored at $-70^{\circ} \mathrm{C}$ until time of biomarker analysis. This simple procedure had high rates of compliance for proper self-application of the sweat patch [3]. After collection, plasma and sweat neuroimmune biomarkers were detected by recycling immunoaffinity chromatography (RIC) coupled with laser-induced fluorescence at the National Institute of Biomedical Imaging and Bioengineering. This methodology has been used to measure cytokines in plasma and cervical secretions [4], in cord blood and postnatal serum from newborns and more recently in sweat and plasma of healthy premenopausal women and women with depression $[3,5]$. Briefly, sweat analytes are vacuum-extracted from the patch, protein content is normalized before applying the extract to an immunoaffinity column array system [4] and results are verified by mass spectrometry and MALDI-TOF. RIC is a single-antibody system that uses a set of high-performance liquid chromatographic immunoaffinity columns in series, with each column immunospecific for a targeted, fluorescently labeled analyte [4]. The advantage of this methodology is that it is highly sensitive and specific and allows measurements of a much larger number of analytes per unit of fluid than are detectable with others methods. In addition, minute volumes of fluid can be analyzed (e.g. a specimen volume of only $25 \mu \mathrm{l}$ is required). One disadvantage of the RIC technology is that it is labor-intensive and therefore not easily applicable in a clinical lab setting. Studies are ongoing to validate other more clinically accessible, high-throughput and sensitive methodologies for analyte quantification. 
In our initial validation studies, we showed that immune biomarkers, such as pro-inflammatory cytokines, in sweat were tightly correlated with plasma levels in healthy controls [3]. These results were obtained using RIC and linear regression analysis, which confirmed that sweat levels were highly predictive of plasma levels. In addition, our preliminary data in a population of women with major depressive disorder (MDD) show elevated sweat levels of pro-inflammatory cytokines, sympathetic neuropeptides (neuropeptide-Y [NPY]) and pain-related neuropeptides (substance P [SP], calcitonin gene-related peptide [CGRP]), but decreased parasympathetic (vasoactive intestinal peptide [VIP]) neuropeptides relative to controls, which strongly correlated with plasma levels [5]. This pattern is consistent with a shift in MDD from parasympathetic to sympathetic tone and an underlying proinflammatory state that could account for enhanced susceptibility to conditions known to be comorbidly expressed with MDD, including cardiovascular disease, osteoporosis and diabetes. Moreover, biomarker levels strongly correlated with symptoms of depression and anxiety, indicating functional significance of these biomarker profiles.

\section{Neural Pathway Evaluation: Peripheral Nervous System and Autonomic Nervous System}

The neural stress system consists of the peripheral nervous system and the ANS. Both systems play an important role in the stress response and modulate the immune system at local and regional levels. The peripheral nervous system regulates the immune system at sites of inflammation through the release of neuropeptides that tend to be pro-inflammatory [2]. As mentioned above, a noninvasive method to measure neuropeptides is through collection of sweat. Our previous studies showed that neuropeptides, such as NPY, VIP, SP and CGRP, can be measured in sweat and are tightly correlated with levels in plasma in patients with history of depression [5]. The sympathetic and parasympathetic (vagus nerve, mediated by acetylcholine) branches of the ANS play an important role in the regulation of the cardiovascular system and the immune system $[6,7]$, where the parasympathetic nervous system has been shown to have an anti-inflammatory influence on the immune system [7].

Evaluation of the ANS can also be performed noninvasively through measurement of HRV. HRV is a term that describes variations of both instantaneous heart rate and the interval between consecutive beats. A prominent circadian variation in HRV, with significant increases during the night and decreases during the day, is observed in healthy individuals. Previous studies have shown that this increase in nighttime HRV is blunted by acute stress, as well as conditions such as chronic alcoholism [6]. Decreased HRV, indicative of reduced parasympathetic/vagal tone, is an independent risk factor for morbidity and mortality.

\section{HPA Axis Evaluation}

Salivary cortisol is an easily obtainable biofluid and noninvasive source for evaluating the HPA axis. This methodology has been widely applied in field-related research settings through the development of adequate ambulatory technology to collect saliva. However, it is important to emphasize that the use of this technique has its limitation and the analysis of the data has to take into account the research question. Studies have shown that measuring cortisol after awakening - the 'cortisol awakening response' (CAR) - is considered an indicator of basal HPA axis activity. In addition, CAR is influenced by several factors such as age, gender, stress perception, health status and lifestyle behaviors. In addition, recent studies have shown that several daily measurements are necessary to assess the CAR [8].

\section{Conclusion}

Simultaneous and repeated evaluation of stress-related systems with noninvasive techniques can help elucidate the underlying interactions of the immune system, ANS, and HPA axis, and their role in disease susceptibility and progression of stress-related disorders. We have established a methodology to simultaneously evaluate a large array of neural and immune biomarkers in sweat, collected through commercially available, FDA-approved cutaneous sweat patches. Applying this new methodology together with two already established methods, HRV to evaluate the ANS and salivary cortisol measurements to evaluate the HPA axis, can aid in identifying biomarker patterns associated with disease stage, severity and outcome, as well as treatment efficacy.

Sweat and saliva collection overcome several of the limitations intrinsic to blood collection. These sampling approaches represent a minimally invasive technique, without pain or distress, for measuring neuroimmune and endocrine parameters, respectively. 
Ultimately, these noninvasive methodologies could provide a 'molecular signature' for clinical subtypes within a heterogeneous disorder, to be used for diagnostic and prognostic purposes; to allow for earlier intervention in asymptomatic conditions; for optimization of individualized treatment regimens; for patient moni- toring in remote areas and in large-scale epidemiological settings; for monitoring patients in which invasive methodologies are unfeasible; and to shed light on mechanisms that underly individual vulnerability or resilience to development of stress-related diseases/disorders.

\section{References}

5 Cizza G, et al: Elevated neuroimmune biomarkers in sweat patches and plasma of premenopausal women with major depressive disorder in remission: the POWER study. Biol Psychiatry 2008;64:907-911.

6 Thayer JF: Vagal tone and the inflammatory reflex. Cleve Clin J Med 2009;76(suppl 2): S23-S26.

7 Tracey KJ: Physiology and immunology of the cholinergic anti-inflammatory pathway. J Clin Invest 2007;117:289-296.

8 Fries EL, Dettenborn J, Kirschbaum C: The cortisol awakening response (CAR): facts and future directions. Int J Psychophysiol 2009;72:67-73

9 Webster JI, Tonelli L, Sternberg EM: Neuroendocrine regulation of immunity. Annu Rev Immunol 2002;20:125-163. 